\title{
Cell Surface-Expressed GPI-Anchored Peptides from the CHR Domain of gp41 Are Potent Inhibitors of HIV-1 Fusion +
}

\author{
Aleksandra Maslennikova ${ }^{1, *}$, Dmitriy Komkov ${ }^{1}$, Anastasia Zotova ${ }^{1}$ and Dmitriy Mazurov ${ }^{1,2}$ \\ 1 Cell and Gene Technology Group, Center for Precision Genome Editing and Genetic Technologies for \\ Biomedicine, Institute of Gene Biology RAS, Moscow 119334, Russia; dmitkomserg@gmail.com (D.K.); \\ ashunaeva@gmail.com (A.Z.); dvmazurov@yandex.ru (D.M.) \\ 2 NRC Institute of Immunology FMBA of Russia, Moscow 115478, Russia \\ * Correspondence: a.k.dorogush@gmail.com \\ † Presented at Viruses 2020 - Novel Concepts in Virology, Barcelona, Spain, 5-7 February 2020. \\ Published: 16 June 2020
}

\begin{abstract}
Current antiretroviral therapy efficiently suppresses viral replication but cannot eliminate latent HIV reservoirs. Moreover, the associated high costs, side effects, and drug resistance have stimulated a need for the development of alternative methods of HIV-1/AIDS treatment, such as peptide inhibitors or gene editing. Recently, we have developed Surface Oligopeptide knock-in for Rapid Target Selection (SORTS), a method for the rapid selection of CRISPR/Cas9 gene-edited cells via knock-in of the Flag and HA epitope tags embedded into the shortest GPI-protein, CD52. By targeting the capsid region of the HIV-1 genome, we demonstrate that SORTS can be applied in provirus eradication. However, the cells with inactivated provirus will be susceptible to HIV reinfection. We hypothesized that knocking in one of the peptides from the CHR-domain of gp41, which are known potent inhibitors of HIV-1 fusion, instead of the epitope tag, will provide "postcurable" HIV-1 resistance. While these peptides were extensively studied as soluble substances, their inhibitory effects on HIV after expression on cell surfaces via GPI-anchor are largely unknown. In this study, we established HEK293T/CD4/R5 and Raji/CD4/R5 HIV-1 permissive cell lines that stably expressed one of the gp41 peptides C34, MT-C34, MT-C34-R, and MT34-15D, or alfa-helix mimetics HP23L, p52, and MT-WQ-IDL. For cell surface delivery, the indicated peptides were embedded into the CD52 molecule, and upstream GFP was used to select transformed cells. Using a single-cycle replication assay with the inLuc reporter vector and different Envs, we demonstrated that C34-based GPI-anchored peptides inhibited both cell-free and cell-to-cell HIV-1 infection by at least two orders of magnitude. With the exception of HP23L, the alfa-helix mimetics were less potent inhibitors. Thus, peptides from gp41 associated with lipid rafts and exerted a strong inhibitory activity which can far exceed that determined for soluble peptides, but this should be tested further.
\end{abstract}

Keywords: HIV-1; fusion inhibitor peptides; GPI-anchored peptides; gene editing; CD52

Funding: This work was supported by grant RFBR 18-29-07052 and by grant 075-15-2019-1661 from the Ministry of Science and Higher Education of the Russian Federation

(C) 2020 by the authors. Licensee MDPI, Basel, Switzerland. This article is an open access article distributed under the terms and conditions of the Creative Commons Attribution (CC BY) license (http://creativecommons.org/licenses/by/4.0/). 